\title{
ASK AN EXPERT
}

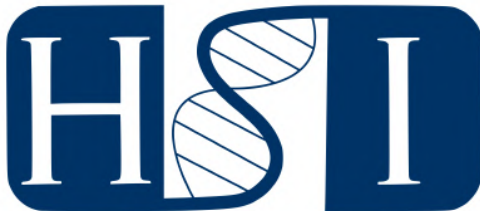

Health Science Inquiry

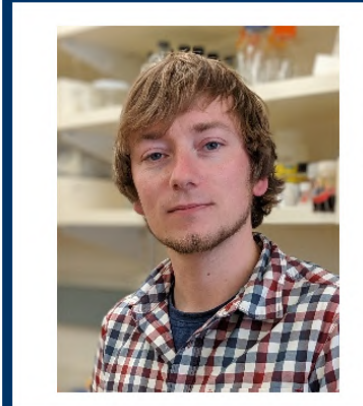

DR. PETER RAHFELD is a scientist with expertise in the fields of high throughput screening and protein biochemistry, working in the Laboratory of Prof. Stephen G. Withers in the Chemistry Department at the University of British Columbia. His research focuses on the discovery of novel carbohydrate active enzymes though functional metagenomic library screenings.

\section{Peter Rahfeld ${ }^{1}$, Stephen Withers ${ }^{1}$}

${ }^{1}$ Department of Chemistry, University of British Columbia

\section{Universal Donor Blood Production: Screening the Human Gut Microbiome for Novel Carbohydrate Active Enzymes}

Blood transfusion is an indispensable part of the health care system, saving thousands of lives annually. Although significant improvements in the collection and use of blood have been made over the years, there are always shortages of matching blood groups. In every blood transfusion and tissue or organ transplantation, the match of the antigens of host and donor are crucial to avoid fatal reactions. The $\mathrm{A}, \mathrm{B}$ and $\mathrm{O}$ carbohydrate antigens of the $\mathrm{ABO}$ blood group system are clinically the most important antigens [1], with about $10^{6}$ antigens present on one cell. The basis of the ABO blood group system is the $\mathrm{O}$ antigen type, which is determined by the $\mathrm{H}$ antigen, a disaccharide moiety attached to glycoproteins and lipids on the surface of red blood cells (RBCs). The A and B antigens are structurally defined through a trisaccharide moiety. Here, the common $\mathrm{H}$ antigen is also decorated with $\alpha$-galactose or $\alpha$ - $\mathrm{N}$-acetylgalactosamine for B-type and A-type red blood cells, respectively (Figure 1). The O-type (H-antigen) cells are non-antigenic for the vast majority of people, and therefore can function as a universal donor group. This leads to a strong demand for blood donations from individuals carrying this blood type, thus often to a shortage.

One method to overcome the shortage of O-type blood is the enzymatic conversion of A, B or AB blood groups to universal donor blood. In this process, the use of glycoside hydrolases, which are enzymes able to remove the $\alpha$-galactose or $\alpha$-N-acetylgalactosamine residues of the trisaccharide moiety present on B- and A-type red blood cells respectively, has emerged as a useful strategy to create O-type blood (H-antigen) (Figure 1). One of the first enzymes discovered to cleave the $\mathrm{B}$ antigens of RBCs was the $\alpha$-galactosidase from coffee bean (Coffea canephora) but harsh reaction conditions and large amounts of protein are necessary to catalyze the reaction, which makes this enzyme unsuitable for general application [2]. Therefore, research has started to focus on the discovery of more efficient enzymes using large scale screenings of bacterial libraries. This has led to the discovery of additional $\alpha$-N-acetylgalactosaminidases and $\alpha$-galactosidases, but unfortunately none of the described enzymes above possess parameters which would allow larger scale industrial application [3]. To truly facilitate large scale conversion of A/B-type RBCs into O-type blood, the universal donor blood, novel highly efficient glycoside hydrolases are urgently needed.

In order to discover novel enzymes for the generation of universal donor blood, we are interested in the enzymes expressed in the human gut microbiome. The human gut mucus layer harbors large glycoproteins, called mucins, presenting a variety of O-glycan structures on their surface, some of them being A, B and $\mathrm{H}$ antigens [4]. Bacteria of the gut microbiome derive energy by foraging those glycans/antigens, and should possess enzymes capable of blood antigen degradation. Utilizing the classic cultivation-based approach would only allow us to scratch the surface of the enzymatic diversity present in the gut microbiome, as $95 \%$ of microbial organisms are uncultivable in laboratory conditions. 

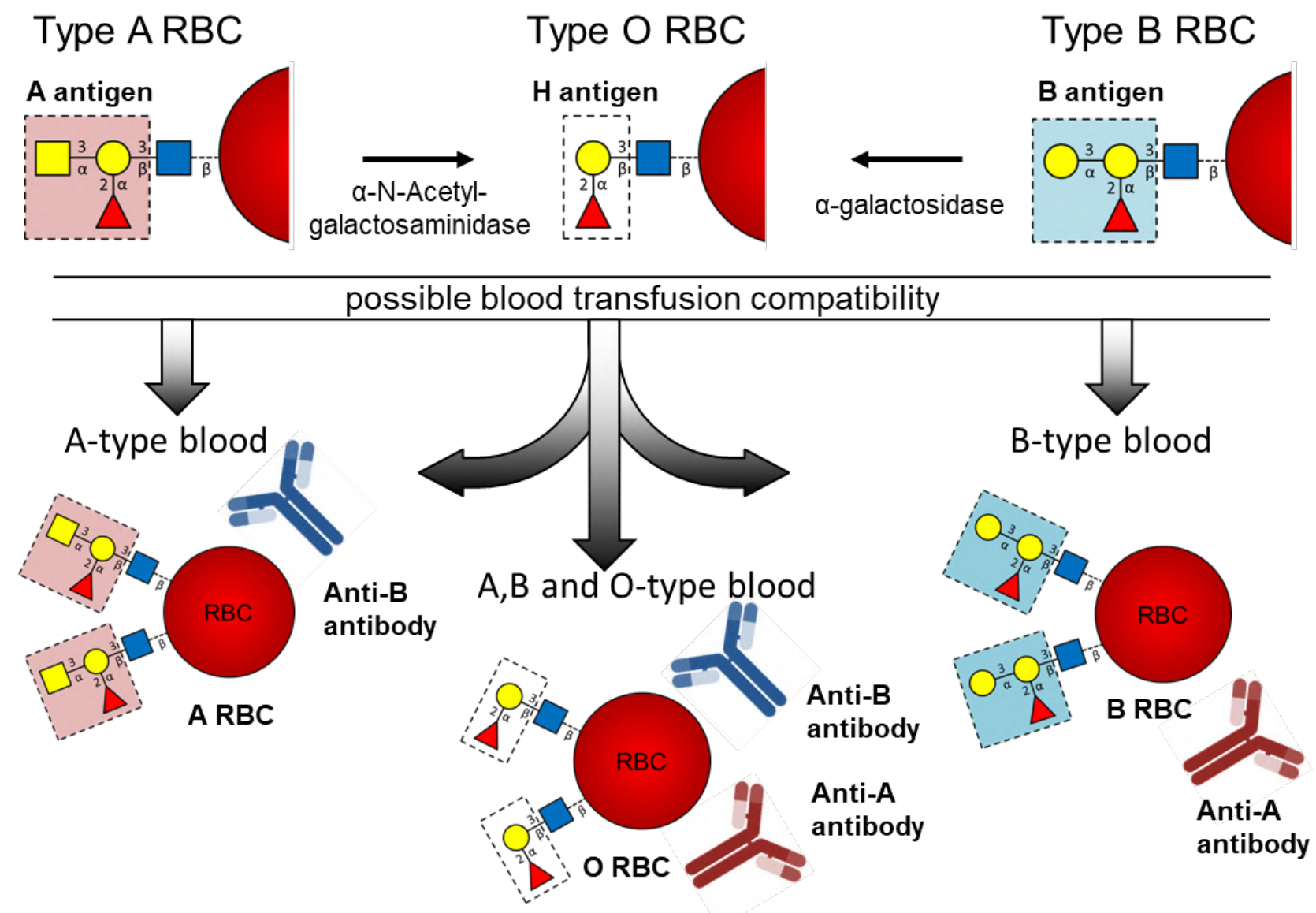

Galactose $\square$ N-Acetylglucosamine

Figure 1: Overview of basic A, B and $\mathrm{H}$ antigens on the surface of Type A,B and O RBCs, and their enzymatic conversion by $\alpha$-N-acetyl-galactosaminidase or $\alpha$-galactosidase to $\mathrm{H}$ antigens, respectively Type O RBCs. Additionally, the blood transfusion compatibility is presented, where only blood without the matching Anti A or B antibody can be mixed. O RBCs and ECO O RBCs are the only ones without any recognized blood group antigen, and therefore are universal donor blood.

Metagenomics, a state-of-the-art technique which can be used to identify and characterize genes and enzymes in complex environmental samples provide a solution [5]. The production of a metagenomic library starts with the isolation of genomic DNA, in this case from human fecal samples which contain microorganisms from the gut. Size-selected genomic DNA is then transferred into a suitable host organism, here Escherichia coli, with the hope of expressing the genes therein. The generated library can be screened for the desired enzyme activity in an approach called functional metagenomic screening, thereby allowing the discovery of novel enzyme activities within a microbiome. To identify blood antigen cleaving enzymes, the human gut library is screened against highly sensitive fluorescent substrates, mimicking the A and B antigens. This functional metagenomic screening yields a novel set of enzymes. Serum-type conversion tests with these enzymes on A-type RBC reveal a highly specific and highly active A antigen cleavage activity, outcompeting any known A antigen cleaving enzyme. The enzymes' ability to completely convert A to O-type RBCs at very low enzyme concentrations in a blood bag turns those enzymes into highly valuable tools to be incorporated into the existing blood transfusion practice to supply universal donor blood. The method of targeted metabolomic screening is easily adaptable to other interesting microbiomes and will expand the access to other important biomedical toolboxes.

\section{REFERENCES}

[1] Daniels G, Reid ME. Blood groups: the past 50 years. Transfusion. 2010;50(2):281-289. 
[2] Goldstein J, Siviglia G, Hurst R, Lenny L, Reich L. Group B erythrocytes enzymatically converted to group O survive normally in A, B, and O individuals. Science. 1982;215(4529):168-170.

[3] Liu QP, Sulzenbacher G, Yuan H, Bennett EP, Pietz G, Saunders K, et al. Bacterial glycosidases for the production of universal red blood cells. Nature biotechnology. 2007;25(4):454.

[4] Tailford LE, Crost EH, Kavanaugh D, Juge N. Mucin glycan foraging in the human gut microbiome. Frontiers in genetics. 2015;6:81.

[5] Handelsman J. Metagenomics: application of genomics to uncultured microorganisms. Microbiol Mol Biol Rev. 2004;68(4):669-685. 